\title{
A New Three-Layer Model for Horizontal Slurry Flow
}

\author{
$1^{\text {st }}$ Alireza Sarraf Shirazi \\ Department of Mechanical Engineering \\ University of British Columbia \\ Vancouver, BC, Canada \\ alireza.sarrafshirazi@gmail.com
}

\author{
$2^{\text {nd }}$ Ian Frigaard \\ Department of Mechanical Engineering \\ University of British Columbia \\ Vancouver, BC, Canada \\ frigaard@math.ubc.ca
}

\begin{abstract}
A modified three-layer model for solid-liquid flow in horizontal pipes is developed, which overcomes the limitations of many previous models. The steady-state model predicts the pressure loss, critical velocity, concentration profile in the heterogeneous layer, mean heterogeneous layer and moving bed layer velocities, and bed layer heights for each set of parameters. We propose a new correlation for the turbulent solids diffusivity. This and the steady state model predictions show a good agreement with experimentally measured results in literature: for concentration profile in the heterogeneous layer and pressure loss, over a wide range of conditions [1]. In turbulent flow. the pressure loss vs mean velocity curve shows a characteristic minimum just before the critical velocity is attained.
\end{abstract}

Index Terms-three-layer model, solids transport, critical velocity, solids diffusivity, pressure gradient

\section{INTRODUCTION}

The flow of solid-liquid mixtures in the form of slurry occurs in a wide range of situations, e.g. river bed erosion and sedimentation, ocean-bed avalanches, transport of mined particulate, etc.. Pipe flows of water-sand slurry are commonly encountered in many applications such as mined slurry transport, hole cleaning, hydraulic fracturing and gravel packing in oil \& gas wells. There are numerous studies of the fundamental sub-processes of solids phase transport (erosion, dispersion, sedimentation, deposition, shear-migration) appearing over the past 20-30 years, and dating back to the 1950s.

Many different two-layer multi-phase hydraulics models have appeared in the slurry transport literature, e.g. the twolayer model of Shook and Bartosik [2], and many others, where a heterogeneous layer, and a sliding/stationary bed layer is predicted. Doron and Barnea [3] first introduced a three-layer slurry transport model. They identified the flow patterns of slurries flowing in horizontal pipes as the flow rate increases: (a) flow with a three-layer configuration, i.e. a stationary bed layer at the bottom, a sliding bed layer in the middle, and a heterogeneous layer at the top; (b) flow with a sliding bed and heterogeneous layer; (c) fully-suspended flow.

Transitions between the different observed flow regimes have historically formed one major axis of the research work on slurry transport. These are typically represented as transition velocities, one of the most important being the deposition, suspension or critical velocity: defining the onset of a bed at the bottom of the pipe. There are many correlations and models for predicting the deposition velocity in literature, e.g. that of Oroskar and Turian [4]. The empirical correlations and theoretical predictions are in qualitative agreement and what is interesting about the methodology in [4] is that it is based on modeling the physical balance between turbulent eddies suspending the particles and buoyancy driven settling.

Other critical velocity predictions in literature are based on a frictional pressure drop prediction, e.g. see [3]. Prediction of frictional pressure gradient is of key importance for industrial applications and has attracted many researchers since 1950's. For example, Turian and Yuan [5] developed correlations based on over 2800 data points, for the frictional pressure in each of the four different flow regimes. This is probably the most comprehensive empirical correlation developed to date.

Particle turbulent diffusivity prediction plays an important role in modeling the slurry flow and predicting the concentration distribution in the pipe. We mention here the work of Walton [6] who proposed a correlation to predict the mean particle diffusivity which consists of three empirical parameters, Based on which he derived an equation for the critical particle velocity. However, the correlation does not account for the dependence of the mean particle diffusivity on the solids concentration.

In the present paper we present a new three-layer model which is based on Doron and Barnea's concept [3], with the main change in defining the solids diffusivity. This affects the prediction of the concentration distribution in the hetero- 
geneous layer and with this the critical velocity. A number of the stress and force closure models in the bed layer were changed and the heterogeneous layer Reynolds number for fine particles was modified. Validation against experimental data is presented in [1]. In $\$[$ II we outline the model development and assumptions, and in \$III, we bring an example of reference outputs from the proposed model.

\section{Model DEVElopment}

In this study we outline a steady state three-layer model used to predict the transport of solids in horizontal wells, typically sand-water flows. These flows arise in many well operations and the model is developed to overcome limitations of existing three- and two-layer models. Conservation of mass equations for the two phases and momentum balance in each layer are combined in the model. A convection-diffusion equation equation models the solids distribution profile and average concentration in the heterogeneous suspension layer, above the bed. These 5 equations plus the imposed flow rate are solved iteratively to predict pressure drop, individual layer heights, mean layer velocities and solids distribution. Additional closure expressions are used to describe other effects.

\section{A. Dimensional analysis}

Steady state slurry flow along the pipe depends on many parameters such as the pipe diameter, $\hat{D}$, the liquid phase density, $\hat{\rho}_{l}$, the solids phase density, $\hat{\rho}_{s}$, the liquid phase viscosity, $\hat{\mu}_{l}$, the particle diameter in the solids phase, $\hat{d}_{p}$, Gravitational acceleration, $\hat{g}$, the flow rate of the slurry, $\hat{Q}$, measured positive in the downwards direction along the pipe, and the fraction of the flow rate due to the solids phase, $q$, (or alternatively, the mean volumetric concentration of solids in pipe cross section, say $C_{v}$ ). The last two mentioned parameters are dimensionless, but others are dimensional 1 Thus, the base flow is described by four dimensionless groups, plus the solids phase flux fraction, $q$, (or $C_{v}$ ). There are various equivalent choices for the four dimensionless groups, but we will adopt two geometric groups, which are the scaled diameter ratio, $\delta=\hat{d}_{p} / \hat{D}$, and the density ratio, $s=\hat{\rho}_{s} / \hat{\rho}_{l}$, and two others, the Froude number $(F r)$ and Reynolds number $(R e)$ :

$$
\begin{aligned}
& R e=\frac{\hat{\rho}_{l} \hat{D} \hat{U}_{s}}{\hat{\mu}_{l}}, \\
& F r=\frac{\hat{U}_{s}^{2}}{\hat{g} \hat{D}(s-1)},
\end{aligned}
$$

where $\hat{U}_{s}=4 \hat{Q} /\left(\pi \hat{D}^{2}\right)$. Other choices could have included a Richardson or Bagnold number.

The base set of five parameters $(R e, s, F r, \delta, q)$ is clearly not sufficient to fully describe all phenomena one is likely to encounter in a pipe flow. Characterising the particle distribution via a single parameter is considered as a simplification, apart from a size distribution particle shape can have significant effects and as soon bed formation and motion

\footnotetext{
${ }^{1}$ Throughout this paper we write all dimensional quantities with a $\hat{.}$ symbol and dimensionless parameters without.
}
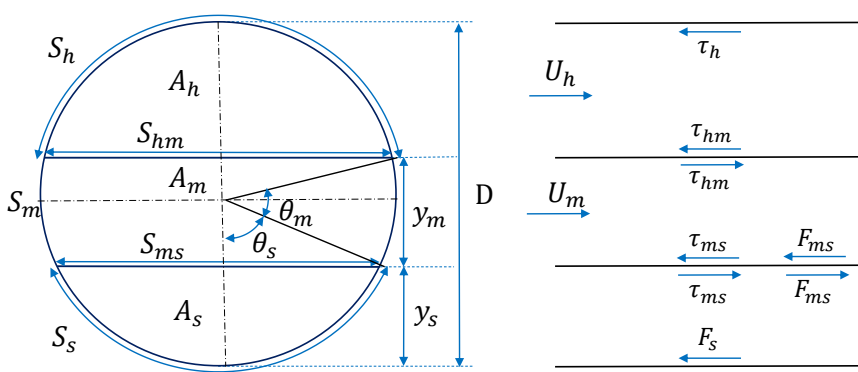

Fig. 1. Schematic of the proposed three-layer model, including geometrical parameters, moving and static bed layers positions, and stresses and forces on each layer

is considered other mechanical and geometric parameters become important, e.g. friction coefficient, maximal packing fraction, etc.

In order to render the model dimensionless we scale all lengths with $\hat{D}$, areas with $0.25 \pi \hat{D}^{2}$, velocities with $\hat{U}_{s}$. The stresses are scaled with $0.5 \hat{\rho}_{l} \hat{U}_{s}^{2}$, forces are scaled with $0.5 \hat{\rho}_{l} \hat{U}_{s}^{2} \hat{D}$ and the pressure gradient is scaled as follows:

$$
\frac{\partial \hat{p}}{\partial \hat{z}}=\left[\hat{\rho}_{l}\left(1-C_{m}\right)+\hat{\rho}_{s} C_{m}\right] \hat{g}+\frac{2 \hat{\rho}_{l} \hat{U}_{s}^{2}}{\pi \hat{D}} \frac{\partial p}{\partial z},
$$

where $0.25 \pi \hat{D}^{2} C_{v}=\hat{A}_{h} C_{h}+\hat{A}_{b} C_{\max }$, i.e. $C_{v}$ is the mean spatial solids concentration.

\section{B. Three-layer steady state model equations}

According to the flow parameters, the pipe cross-section may contain moving and/or stationary beds at the bottom of the pipe, of heights $\hat{y}_{m}$ and $\hat{y}_{s}$ respectively, see Fig. 1 . Above the bed the flow is assumed to consist of a heterogeneous slurry, in which the concentration (volume fraction) of solids varies with height $\hat{y}_{h}$. Within the bed the concentration is assumed to be at the maximal packing fraction, $C=C_{\max }$. The heterogeneous layer moves in the axial direction with mean speed $\hat{U}_{h}$ and the moving bed layer moves with mean speed $\hat{U}_{m}$.

Conservation of the total mass flow rate is represented by

$$
\hat{Q}=0.25 \pi \hat{D}^{2} \hat{U}_{s}=\hat{A}_{h} \hat{U}_{h}+\hat{A}_{m} \hat{U}_{m},
$$

and mass conservation of the solid and liquid phases is governed by:

$$
\begin{aligned}
\hat{Q} q & =C_{h} \hat{A}_{h} \hat{U}_{h}+C_{\max } \hat{A}_{m} \hat{U}_{m} \\
\hat{Q}(1-q) & =\left(1-C_{h}\right) \hat{A}_{h} \hat{U}_{h}+\left(1-C_{\max }\right) \hat{A}_{m} \hat{U}_{m}
\end{aligned}
$$

where $C_{h}$ is the mean solids concentration of the heterogeneous layer and the areas $\hat{A}_{h}$ and $\hat{A}_{m}$ are heterogeneous and moving bed cross section areas respectively. Evidently, one of Eqs. [3- 5] is redundant.

We select Cartesian coordinates such that $\hat{z}$ denotes axial distance along the pipe in the downwards direction, $\hat{y}$ measure distance perpendicularly upwards from the base of the pipe, 
viewed in a cross-section, and the $\hat{x}$ direction is orthogonal, within the plane of the cross-section. The axial momentum balance in heterogeneous and moving bed layers are:

$$
\begin{aligned}
& \hat{A}_{h} \frac{\partial \hat{p}}{\partial \hat{z}}=-\hat{S}_{h} \hat{\tau}_{h w}-\hat{S}_{h m} \hat{\tau}_{h m} \\
& \hat{A}_{m} \frac{\partial \hat{p}}{\partial \hat{z}}=-\hat{F}_{m w}-\hat{F}_{m s}-\hat{S}_{m s} \hat{\tau}_{m s}-\hat{S}_{m} \hat{\tau}_{m w}+\hat{S}_{h m} \hat{\tau}_{h m}
\end{aligned}
$$

where the perimeters $\hat{S}_{h}$ and $\hat{S}_{m}$ are illustrated in Fig. 1] The axial pressure gradient is denoted $\frac{\partial \hat{p}}{\partial \hat{z}}$. The mean shear stresses along $\hat{S}_{h w}$ and $\hat{S}_{m w}$ are denoted $\hat{\tau}_{h w}$ and $\hat{\tau}_{m w}$, respectively. $\hat{F}_{m s}$ is the dry friction force acting at the interface between the moving bed and stationary bed, $\hat{\tau}_{m s}$ is the hydrodynamic shear stress acting on the interface. $\hat{F}_{m w}$ is the dry friction force acting at the surface of contact of the moving bed with the pipe wall, $\hat{S}_{m w}$, and $\hat{\tau}_{m w}$ is the hydrodynamic shear stress acting on the pipe wall. Closure models for the stresses and forces are described in \$I-D

To obtain the velocity of the moving bed layer, which is a key feature of the three-layer model, we use the method suggested by [3], in which a moment balance equation is written on a solid particle in the lowermost stratum of the moving bed layer, which is at the verge of rolling (for more details, see [3]). Evaluating the moments exerted about the particle, just about to move, we get:

$$
\hat{U}_{m}=\sqrt{\frac{0.779\left(\hat{\rho}_{s}-\hat{\rho}_{l}\right) \hat{g} \hat{d}_{p}\left[C_{\max } \frac{\hat{y}_{m}}{\hat{d}_{p}}+\left(1-C_{\max }\right)\right]}{\hat{\rho}_{l} C_{D}}} .
$$

Within the heterogeneous layer, the solids distribution $C(\hat{y})$ is governed by a balance between sedimentation and turbulent resuspension:

$$
0=\frac{\partial}{\partial \hat{y}}\left[\hat{v}_{p} C+\hat{\epsilon}_{p} \frac{\partial C}{\partial \hat{y}}\right]
$$

where $\hat{v}_{p}$ is the sedimentation velocity and $\hat{\epsilon}_{p}$ is the turbulent particle diffusivity. At the interface between bed and heterogeneous layer we have $C=C_{\max }$, and at the top of the heterogeneous layer there is no flux of particles, so that:

$$
\hat{v}_{p} C+\hat{\epsilon}_{p} \frac{\partial C}{\partial \hat{y}}=0 .
$$

\section{Concentration distribution in the heterogeneous layer}

On scaling $\hat{y}$ with $\hat{D}$, and on integrating (9), the solids distribution within the heterogeneous layer $\left(y_{b}, 1\right]$ satisfies the following initial value problem:

$$
\frac{\partial C}{\partial y}+\frac{\hat{v}_{p} \hat{D}}{\hat{\epsilon}_{p}} C=0, \quad\left(y_{b}, 1\right], \quad C\left(y_{b}\right)=C_{0} .
$$

In the case that there is a bed, $\left(y_{b}>0\right)$, then the initial condition is given by $C_{0}=C_{\max }$. The differential equation
(11) is solved to give $C(y)$ and the area-averaged value of $C(y)$ is then computed as follows:

$$
\bar{C}\left(y_{b}\right)=\frac{\int_{y_{b}}^{1} C(y) \sqrt{1-(2 y-1)^{2}} \mathrm{~d} y}{\int_{y_{b}}^{1} \sqrt{1-(2 y-1)^{2}} \mathrm{~d} y},
$$

We note that the value of $\bar{C}$ depends also on the initial condition $C_{0}$, used in (11), and on any other dimensionless parameters in 11 . Thus, we write $\bar{C}=\bar{C}\left(y_{b}, C_{0}\right)$ and the height of the heterogeneous layer is determined from the equation:

$$
\bar{C}\left(y_{b}, C_{0}=C_{\max }\right)=C_{h} .
$$

In the case that there is no bed, $\left(y_{b}=0\right)$, then the initial condition is unknown but the average concentration must still equal $C_{h}$. Thus, $C(y)$ satisfies $\sqrt{11}$ and the initial condition is found from

$$
\bar{C}\left(y_{b}=0, C_{0}\right)=C_{h} .
$$

Evidently, the solution of (11) depends on the closure laws used for the sedimentation velocity, $\hat{v}_{p}$, and for the turbulent particle diffusivity, $\hat{\epsilon}_{p}$.

1) Sedimentation velocity, $\hat{v}_{p}$ : The sedimentation velocity is assumed to depend on the particle concentration according to Richardson and Zaki's hindered settling law [7]:

$$
\hat{v}_{p}=\hat{v}_{p 0}(1-C)^{n},
$$

where $\hat{v}_{p 0}$ is the settling velocity of a single particle and $n$ depends upon the particle Reynolds number, $R e_{p}$. The settling velocity of a single particle could be obtained by a force balance between the gravitational and drag forces:

$$
\hat{v}_{p 0}=\sqrt{\frac{4 \hat{g}(s-1) \hat{d}_{p} \sin \beta}{3 C_{D}\left(R e_{p}\right)}},
$$

where $C_{D}\left(R e_{p}\right)$ is the drag coefficient:

$$
C_{D}\left(R e_{p}\right)= \begin{cases}\frac{24}{R e_{p}} & R e_{p}<1.4 \\ \frac{A_{C D}}{R e_{p}^{0.625}} & 1.4 \leq R e_{p} \leq 500 \\ \frac{A_{C D}}{500^{0.625}} & 500<R e_{p}\end{cases}
$$

where $A_{C D}=24 / 1.4^{0.375}$, and

$$
R e_{p}=\frac{\hat{\rho}_{l} \hat{v}_{p 0} \hat{d}_{p}}{\hat{\mu}_{l}} .
$$

Using the groups $R e, F r$, and $\delta$, we can solve explicitly for $\hat{v}_{p 0}$, from which $R e_{p}$ is determined.

For very low particle Reynolds numbers, the exponent $n$ only depends on the diameter ratio $\delta$, whereas in the intermediate range of Reynolds number it depends on both $\delta$ and Reynolds number, and lastly, for high Reynolds numbers it is a constant. These closure expressions can be found in [7]. It is also worth mentioning that the range of $R e_{p}>500$ is unlikely to be attained. The values of $n$ are between 2.39 
for inertia dominated settling and 4.65 for viscous dominated settling, and also it is a strong function of $R e_{p}$ specially when $0.2 \leq R e_{p}<500$, while it is a weak function of $\delta$.

2) Turbulent particle diffusivity, $\hat{\epsilon}_{p}$ : For obtaining the solids diffusivity, we have adopted and modified Eskins model [8], which is based on an expression for the turbulent diffusivity in isotropic turbulence and is characterized by two empirical parameters that have been identified from the experimental data for solids concentration distribution available in the literature. Eskins model only covers a small range of diameter ratio $\left(\delta=3.636 e^{-4}\right.$ to $\left.9.320 e^{-3}\right)$, and also used the pressure drop model of Turian and Yuan [5] to obtain the pressure drop and the Fanning friction factor along the pipe. Here we have modified the solid diffusivity model taking into account a wider range of diameter ratio including much courser ones, using the pressure drop obtained by the present model. The modified solids diffusivity correlation is validated against the experimental data for solids concentration distribution and critical velocity in the literature; see [1]. The modified solid diffusivity correlation is as follows.

$$
\hat{\epsilon}_{p}=\alpha \delta^{\zeta}\left(\frac{\overline{\hat{\rho}}_{h}}{\hat{\rho}_{h}} \frac{d p}{d x}\right)^{\frac{2}{3}}\left(1+\frac{\tau_{s}}{T_{l}}\right) \hat{D} \hat{U}_{s},
$$

where $\overline{\hat{\rho}}_{h}$ is the mean slurry density in the heterogeneous layer, $\hat{\rho}_{h}$ is the slurry density which is a function of the local concentration, $\tau_{s}$ is the particle relaxation time, and $T_{l}$ is the Lagrangian time scale for the pipe flow. This model was fitted against the experimental concentration data using least square method and we obtained $\alpha=0.81$, and $\zeta$ was found to be dependent on the diameter ratio as

$$
\zeta=-333.3 \delta^{2}+26.37 \delta+1.355 .
$$

The particle relaxation time can be calculated as

$$
\tau_{s}=\tau_{s t} \frac{24}{R e_{p} C_{D}}
$$

where $\tau_{s t}$ is the Stokesian particle residence time in the flow.

\section{Closure equations for stresses and forces}

In the following subsections we define the various closures we have used for the stresses and forces in each layer. For friction factors we use the Fanning friction factor as opposed to the Darcy-Weisbach friction factor, i.e. the relation between a wall stress $\hat{\tau}_{w}$ and the friction factor $f$ is

$$
\hat{\tau}_{w}=0.5 f \hat{\rho} \hat{U}^{2},
$$

and typically $f=16 / R e$ in laminar flow. Explicit friction factor formulas will be defined in the following subsections for each layer.

1) Heterogeneous layer: The wall shear stress in the heterogeneous layer is defined by

$$
\hat{\tau}_{h w}=0.5\left[\hat{\rho}_{s} C_{h}+\hat{\rho}_{l}\left(1-C_{h}\right)\right] \hat{U}_{h}\left|\hat{U}_{h}\right| f\left(R e_{h}, 0\right),
$$

where $R e_{h}$ is the heterogeneous layer Reynolds number and $f_{C W}$ is the friction factor, based on the Colebrook-White relation for turbulent flows, as described below.
We base the heterogeneous layer Reynolds number $R e_{h}$ on the hydraulic diameter of the layer, on $\hat{U}_{h}$, on the mean density of the slurry and on an effective viscosity. Typically these flows are fully turbulent and the viscosity to use depends therefore on how viscous stresses are conveyed to the wall. In the case that the particles are larger than the viscous sub-layer in the flow, then the fluid effective viscosity is simply $\hat{\mu}_{l}$. In the case that the particle size is comparable to the thickness of the viscous sub-layer, we treat the slurry as a pseudo-fluid, with effective viscosity:

$$
\hat{\mu}_{s}=\hat{\mu}_{l}\left[1.0+2.5 C_{h}+10 C_{h}^{2}+0.0019 \mathrm{e}^{20 C_{h}}\right],
$$

see [9]. Eq. (23) is an extension of the Einstein-Thomas relation to higher concentrations and sand particles.

To estimate the size of the viscous sublayer $\hat{d}_{v}$ we use the estimate

$$
\hat{d}_{v} \approx 10 \frac{\hat{D}_{h}}{R e_{h} \sqrt{0.5 f\left(R e_{h}, 0\right)}}
$$

(i.e. the viscous layer is approximately $10 y^{+}$in turbulent duct flows). Here $\hat{D}_{h}$ is the hydraulic diameter of the heterogeneous layer and $f$ is the friction factor.

If the particle diameter $\hat{d}_{p}$ satisfies $\hat{d}_{p}<\hat{d}_{v}$ we assume 23. to be valid: $\hat{\mu}_{h}=\hat{\mu}_{s}$. If the particle diameter is significantly larger than $\hat{d}_{v}$, say $\hat{d}_{p}>10 \hat{d}_{v}$, we assume that $\hat{\mu}_{h}=\hat{\mu}_{l}$. For intermediate values of $\hat{d}_{p} / \hat{d}_{v}$ we interpolate between the above values. Finally, $R e_{h}$ could be obtained using the effective viscosity in the heterogeneous layer, $\hat{\mu}_{h}$. We note that the viscosity ratio $\hat{\mu}_{h} / \hat{\mu}_{l}$ and $R e_{h}$ depend on the 6 parameters $\left(R e, C_{h}, s, \delta, y_{b}, u_{h}\right)$.

The friction factor formula we use is based on the Colebrook-White equation for turbulent flow. The ColebrookWhite expression is:

$$
\sqrt{1 / f}=-2 \log \left(\frac{2.51}{R e \sqrt{f}+\frac{\epsilon_{r}}{3.71}}\right),
$$

which gives a Darcy-Weisbach friction factor $f\left(R e, \epsilon_{r}\right)$.

2) Interfacial stresses: The interfacial stresses are defined in essentially the same as the heterogeneous layer stresses, except that the velocity differences are used, and a roughness is assumed at each interface to determine the corresponding friction factors.

We assume that the "interface" between the heterogeneous and moving bed layers is rough, with the roughness $\epsilon_{r}=$ $\min \left\{1, \delta / d_{h}\right\}$ where $d_{h}$ is the dimensionless hydraulic diameter of the heterogeneous layer. We also assume the "surface" between heterogeneous layer and bed to be "rough", with roughness $\epsilon_{r}=\min \left\{1, \delta / d_{h}\right\}$. The interfacial stress is then defined as follows:

$$
\begin{aligned}
\hat{\tau}_{h m} & =0.5\left[\hat{\rho}_{s} C_{h}+\hat{\rho}_{l}\left(1-C_{h}\right)\right]\left(\hat{U}_{h}-\hat{U}_{m}\right) \\
& \times\left|\hat{U}_{h}-\hat{U}_{m}\right| f\left(R e_{h}, \min \left\{1, \delta / d_{h}\right\}\right) .
\end{aligned}
$$

Similarly, for the interface between the moving and stationary bed layers, we assume the roughness $\epsilon_{r}=\min \left\{1, \delta / d_{m}\right\}$, and accordingly, we define the dimensional interface stress as follows. 


$$
\begin{aligned}
\hat{\tau}_{m s} & =0.5\left[\hat{\rho}_{s} C_{\max }+\hat{\rho}_{l}\left(1-C_{\max }\right)\right] \hat{U}_{m}\left|\hat{U}_{m}\right| \\
& \times f\left(\operatorname{Re}_{h}, \min \left\{1, \delta / d_{m}\right\}\right),
\end{aligned}
$$

where $d_{m}$ is the dimensionless hydraulic diameter of the moving bed layer. In addition to the defined interfacial stresses in 26], we should consider the solid particle contribution to the friction force on the interface $S_{m s}$ due to the effect of the submerged weight of the particles in the moving bed layer. In the heterogeneous layer, it is assumed that the weight of the particles is entirely supported by turbulent suspension. In contrast, the submerged weight of the particles in the moving bed layer is supported by the wall and also the interface $S_{m s}$. The resulting dry friction force at the interface which is transmitted to the wall as a Coulomb friction term is defined as

$$
\hat{F}_{m s}=\eta\left(\hat{\rho_{s}}-\hat{\rho}_{l}\right) g C_{\max } \hat{y_{m}} \hat{S_{m s}} .
$$

3) moving bed layer: Our treatment of the moving bed layer wall shear stress is similar to that of the heterogeneous layer, which is defined as

$$
\begin{aligned}
\hat{\tau}_{m w} & =0.5\left[\hat{\rho}_{s} C_{\max }+\hat{\rho}_{l}\left(1-C_{\max }\right)\right] \hat{U}_{m}\left|\hat{U}_{m}\right| \\
& \times f\left(R e_{m}, 0\right) .
\end{aligned}
$$

Where $R e_{m}$ is the moving bed layer Reynolds number which could be determined as follows.

$$
R e_{m}=\operatorname{Re} d_{m}\left|u_{m}\right|\left(1+(s-1) C_{\max }\right) .
$$

As was discussed in \$II-D2 the submerged weight of the solid phase is partially transmitted through the bed to the wall as a Coulomb friction term. Thus, the dimensional term of the dry friction force at the wall is defined as follows.

$$
\begin{aligned}
& \hat{F}_{m w}=2 \eta\left(\hat{\rho}_{s}-\hat{\rho}_{l}\right) g C_{\max }\left(\frac{D}{2}\right)^{2} \\
\times & \left\{\left[2 \frac{\left(\hat{y}_{s}+\hat{y}_{m}\right)}{D}-1\right] \theta_{m}+\cos \left(\theta_{m}+\theta_{s}\right)-\cos \theta_{s}\right\} .
\end{aligned}
$$

4) Static bed layer, and transition to two-layer solution: The existence of a static bed is determined based on the momentum balance equation on the static bed layer. It should be checked whether the sum of driving forces on the static bed layer exceeds the maximal available resistance force on this layer. The driving forces on the static layer are pressure gradient, and the shear at the interface between the moving and static bed layers. The maximal available resistance is the dry friction force acting on the periphery of the static bed which is defined as follows.

$$
\begin{gathered}
\hat{F}_{m w}=2 \eta_{s}\left(\hat{\rho}_{s}-\hat{\rho}_{l}\right) g C_{\max }\left(\frac{D}{2}\right)^{2} \\
\times\left[\left(\frac{2 \hat{y}_{s}}{\hat{D}}-1\right)\left(\theta_{s}+\frac{\pi}{2}\right)+\cos \theta_{s}\right] .
\end{gathered}
$$

where $\eta_{s}$ is the dry static friction coefficient. if

$$
\hat{A}_{s} \frac{\partial \hat{p}}{\partial \hat{z}}+\hat{F}_{m s}+\hat{S}_{m s} \hat{\tau}_{m s} \leqslant \hat{F}_{s w}
$$

is satisfied, the static bed layer does not move, and it is indeed "static"; Otherwise, the static bed layer moves and we get a transition from three-layer model solution to the twolayer model solution, where we assume that there is one bed layer in the pipe which is moving. For solving the two-layer problem, we adopt the similar procedure to the three-layer problem. The only difference between the two problems is that in the two-layer model, five equations are numerically handled to get the five unknowns $\hat{U}_{h}, \hat{U}_{m}, \hat{y}_{m}, C_{h}, \frac{\partial \hat{p}}{\partial \hat{z}}$, whereas for three-layer model we deal with six equations to get six unknowns (aformentioned unknowns plus $\hat{y}_{s}$ ).

\section{RESULT AND DISCUSSION}

An example of reference outputs from the proposed model is given in Figs. 2(a-f). We present results for $C_{v}=$ $0.2,0.25,0.3,0.35$, over a wide range of mean superficial velocities, for the horizontal pipe diameter of $D=0.1 \mathrm{~m}$. For each example we present the solids phase flux fraction, frictional pressure drop, heterogeneous and moving bed layers velocities, and moving and static bed layers heights. Note that all of the outputs in Figs. 2(a-f) are dimensionless except for the pressure drop result (Fig. 2(f)). As mentioned before, the velocities are scaled with slurry mean superficial velocity $\hat{U}_{s}$, and the lengths are scaled with the pipe diameter $D$.

Eq. 11 suggests that the concentration distribution, and the bed height in the pipe depend on the competition between the sedimentation velocity and solid turbulent diffusivity. At low mean superficial velocities, the turbulent eddies are not strong enough to suspend the solid phase; As a result , a considerable portion of the pipe is covered with bed layers, and we get low mean delivered solids concentration (Figs. 2(a), 2(c), and 2(e)). Also, the height of the static bed is larger than the moving bed (Figs. 2(c) and 2(e)). As a result, the mean heterogeneous layer velocity is at its maximum at low flow rates to satisfy the continuity equation (Fig. 2(b)).

As the mean superficial velocity increases, the turbulent eddies get more capable of suspending the solids; Thus, the mean delivered solids concentration increases as could be observed in Fig. 2(a). Furthermore, the height of moving bed increases as the height of static bed drops(Figs. 2(c) and 2 (e)). The total bed height also decreases with increasing the flow rate. From Eq. (8) it is obvious that the mean moving bed velocity $\hat{U}_{m}$ monotonically increases with the moving bed height as long as we get a three-layer configuration with a static bed, although Fig. 2(d) could be misleading as it shows the dimensionless mean moving bed velocity $u_{m}$ decreases with the flow rate increases.

As the flow rate increases, we reach a point where there is no static bed layer, and the pipe consists of the heterogeneous and the moving bed layers. As was discussed in \$II-D4, this is the transition from the three-layer to two-layer model solution, where the mean velocity and the height of the moving bed 

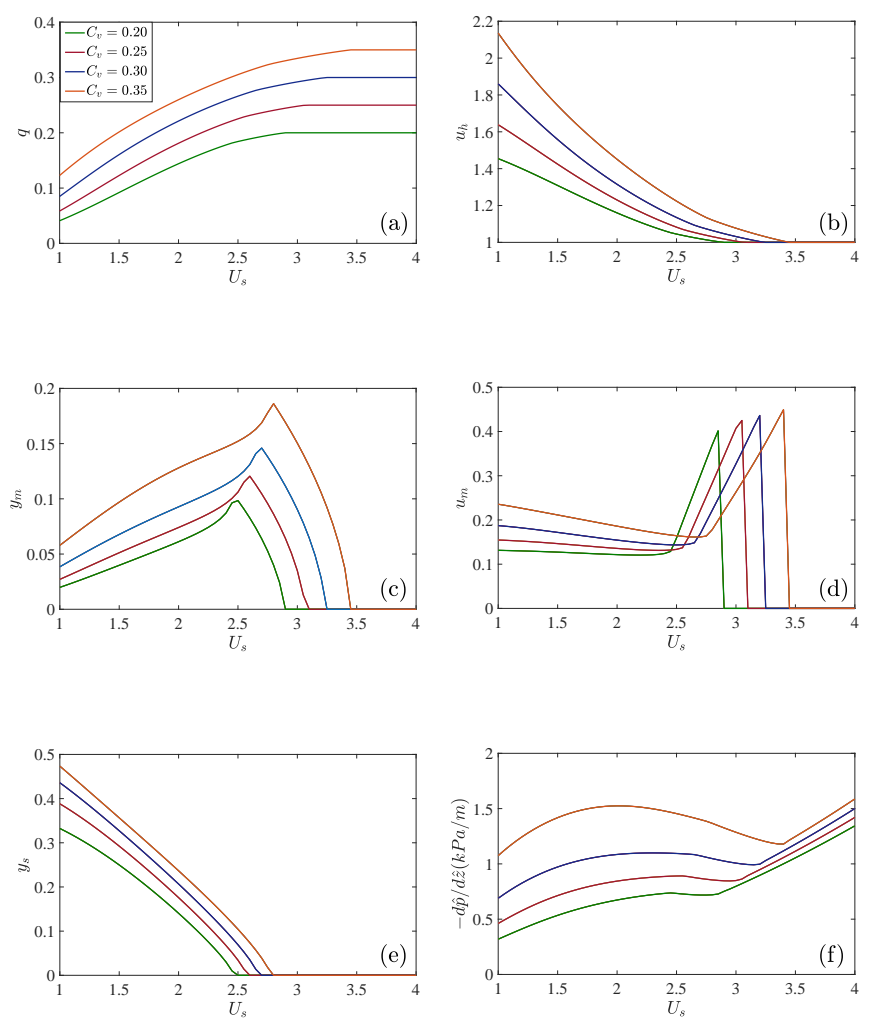

Fig. 2. Example solutions from the proposed steady state three-layer model problem at various $C_{v}$, as a function og $\hat{U}_{s}$, for a sand-water mixture flowing in a horizontal pipe of diameter $\hat{D}=0.1 \mathrm{~m}$. Fixed parameters are $C_{\max }=$ $0.55, \hat{\rho}_{s}=2650 \mathrm{~kg} / \mathrm{m}^{3}, \hat{\rho}_{l}=1000 \mathrm{~kg} / \mathrm{m}^{3}, \hat{\mu}_{l}=9 \times 10^{-4} \mathrm{~Pa} . \mathrm{s}, \hat{d}_{p}=$ $7 \times 10^{-4} \mathrm{~m}, \eta=0.5$, and $\eta_{s}=0.7$.

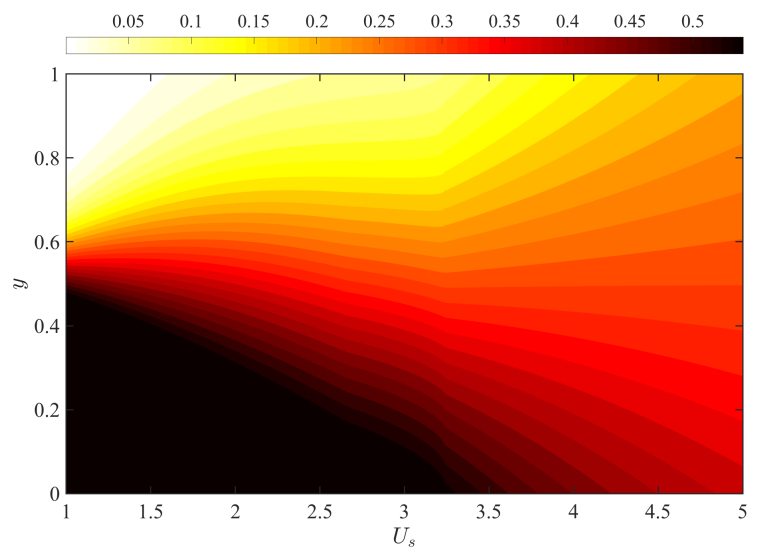

Fig. 3. colormap of concentration in $\left(\hat{U}_{s}, y\right)$ plane for $C_{v}=0.3$. Fixed parameters are $C_{\max }=0.55, \hat{\rho}_{s}=2650 \mathrm{~kg} / \mathrm{m}^{3}, \hat{\rho}_{l}=1000 \mathrm{~kg} / \mathrm{m}^{3}, \hat{\mu}_{l}=$ $9 \times 10^{-4}$ Pa.s, $\hat{d}_{p}=7 \times 10^{-4} \mathrm{~m}, \eta=0.5$, and $\eta_{s}=0.7$. layer reach their maximum values. As we further increase the flow rate, the height of moving bed decreases until we reach the deposition velocity where there is no sedimentation bed, and we get a single heterogeneous layer across the pipe, and evidently, the mean delivered solids concentration is equal to the mean spatial solids concentration (Fig. 22a)). As can be observed in Fig. 2(f), the pressure gradient reaches its minimum value at the deposition velocity. Furthermore, the deposition velocity increases with $C_{v}$. These findings are consistent with the published results of most of the researchers in the field, e.g. [2], [5].

Fig. 3 shows a colormap of solids concentration in the $\left(\hat{U}_{s}, y\right)$ plane for a constant value of $C_{v}=0.3$. At low flow rates, the bottom of the pipe is at maximum packing concentration $C_{\max }$ which indicates the existence of the sedimentation bed. Also, we observe that the concentration gradient is very large in the heterogeneous layer at low flow rates. As $\hat{U}_{s}$ increases, the total bed height decreases as was discussed before, and the concentration gradient drops as well. At very high velocities, the solids turbulent diffusivity becomes dominant and we get a pseudo-homogeneous slurry flow in the pipe.

\section{Conclusions}

A modified three-layer model for solid-liquid flow in horizontal pipes has been developed which predicts the pressure loss, critical velocity, concentration profile in the heterogeneous layer, mean heterogenous layer and moving bed layer velocities, and bed layer heights for each set of parameters. We have also proposed a new correlation for the turbulent solids diffusivity based on concentration profile and critical velocity comparison against the experimental data available in the literature. The pressure loss vs mean velocity curve shows a characteristic minimum just before the critical velocity is attained, in agreement with published research.

\section{REFERENCES}

[1] A. Sarraf Shirazi and I. Frigaard, "A novel three-layer model for steadystate slurry flow in inclined pipes," unpublished.

[2] C. Shook and A. Bartosik, "Particlewall stresses in vertical slurry flows," Powder Technology, vol. 81, no. 2, pp. 117 - 124, 1994.

[3] P. Doron and D. Barnea, "A three-layer model for solid-liquid flow in horizontal pipes," International Journal of Multiphase Flow, vol. 19, no. 6, pp. $1029-1043,1993$.

[4] A. R. Oroskar and R. M. Turian, "The critical velocity in pipeline flow of slurries," AIChE Journal, vol. 26, no. 4, pp. 550-558, 1980.

[5] R. M. Turian and T.-F. Yuan, "Flow of slurries in pipelines," AIChE Journal, vol. 23, no. 3, pp. 232-243, 1977.

[6] I. WALTON, "Eddy diffusivity of solid particles in a turbulent liquid flow in a horizontal pipe," AICHE JOURNAL, vol. 41, no. 7, pp. 1815-1820, 1995.

[7] J. F. Richardson and W. N. Zaki, "Sedimentation and fluidisation: Part i," Chemical Engineering Research and Design, vol. 75, pp. S82-S100, 1997.

[8] D. Eskin, "A simple model of particle diffusivity in horizontal hydrotransport pipelines," Chemical Engineering Science, vol. 82, pp. 84 - 94, 2012.

[9] J. Schaan, R. J. Sumner, R. G. Gillies, and C. A. Shook, "The effect of particle shape on pipeline friction for newtonian slurries of fine particles," The Canadian Journal of Chemical Engineering, vol. 78, no. 4, pp. 717$725,2000$. 